\title{
Cervical spine tuberculosis and airway compromise
}

\author{
B. S. Deepti, MD · Manjunath Munireddy, DA, DNB · Sriganesh Kamath, MD, DM • \\ Dhritiman Chakrabarti, MD
}

Received: 1 December 2015/Revised: 7 December 2015/Accepted: 16 December 2015/Published online: 6 January 2016

(C) Canadian Anesthesiologists' Society 2016

A ten-year-old child presented with neck pain and progressive paraparesis of two months' duration. Magnetic resonance imaging (MRI) of the cervical spine showed a prevertebral abscess collection at the C7-T4 spinal level that was compressing the trachea (Figure). A diagnosis of spinal tuberculosis (i.e., Koch's spine with Pott's paraplegia) was suspected. The patient was scheduled for resection of the prevertebral abscess and posterior cervical spine stabilization via anterior fusion. As the child was uncooperative for awake fibreoptic intubation and both the clinical airway examination and MRI showed that the upper airway anatomy was relatively normal, we chose an inhalational anesthesia induction. After successful mask ventilation was confirmed and following administration of a muscle relaxant, direct laryngoscopy with orotracheal intubation was performed. A $6.5 \mathrm{~mm}$ armoured endotracheal tube (ETT) was secured at $18 \mathrm{~cm}$ depth after confirming equal air entry in both lungs. During decompression of the abscess, retraction was used for exposure, which resulted in an abrupt increase in airway pressure with decreased tidal volume and increased $\mathrm{ETCO}_{2}$ (to $50 \mathrm{mmHg}$ ). Fluoroscopy showed that the retractor was located just below the metallic portion of the armoured ETT, possibly compromising the softer distal ETT and/or the tracheal lumen. The ETT was advanced to $20 \mathrm{~cm}$, and bilateral air entry was reconfirmed, after which ventilation and subsequent surgery were uneventful. A postoperative computed tomography scan revealed good stabilization of the cervical spine. The trachea was extubated $80 \mathrm{hr}$ postoperatively. There was significant postoperative neurological improvement with the lower limb strength, on a scale of 0 to 5 , increasing to a score of 4 .

The spinal column is involved in $<1 \%$ of all cases of tuberculosis (TB). ${ }^{1}$ Cervical spine TB is even more rare, accounting for only 3-5\% of all spinal TB cases. $^{2}$ A tuberculous dorsal cervical prevertebral abscess with edema can distort normal airway anatomy, necessitating fibreoptic intubation. ${ }^{3}$ Unlike a previous report, our patient's intubation was relatively easy, however, the ventilation was subsequently affected because the tip of the armoured ETT was compressed during surgical retraction, necessitating repositioning the ETT beyond the pathological area. Continued vigilance is clearly required not only during intubation but througchout the case.

B. S. Deepti, MD ( $₫) \cdot$ M. Munireddy, DA, DNB ·

S. Kamath, MD, DM - D. Chakrabarti, MD

Department of Neuroanesthesia, National Institute of Mental

Health and Neuro Sciences, Bangalore, India

e-mail: bsdeepti@gmail.com 

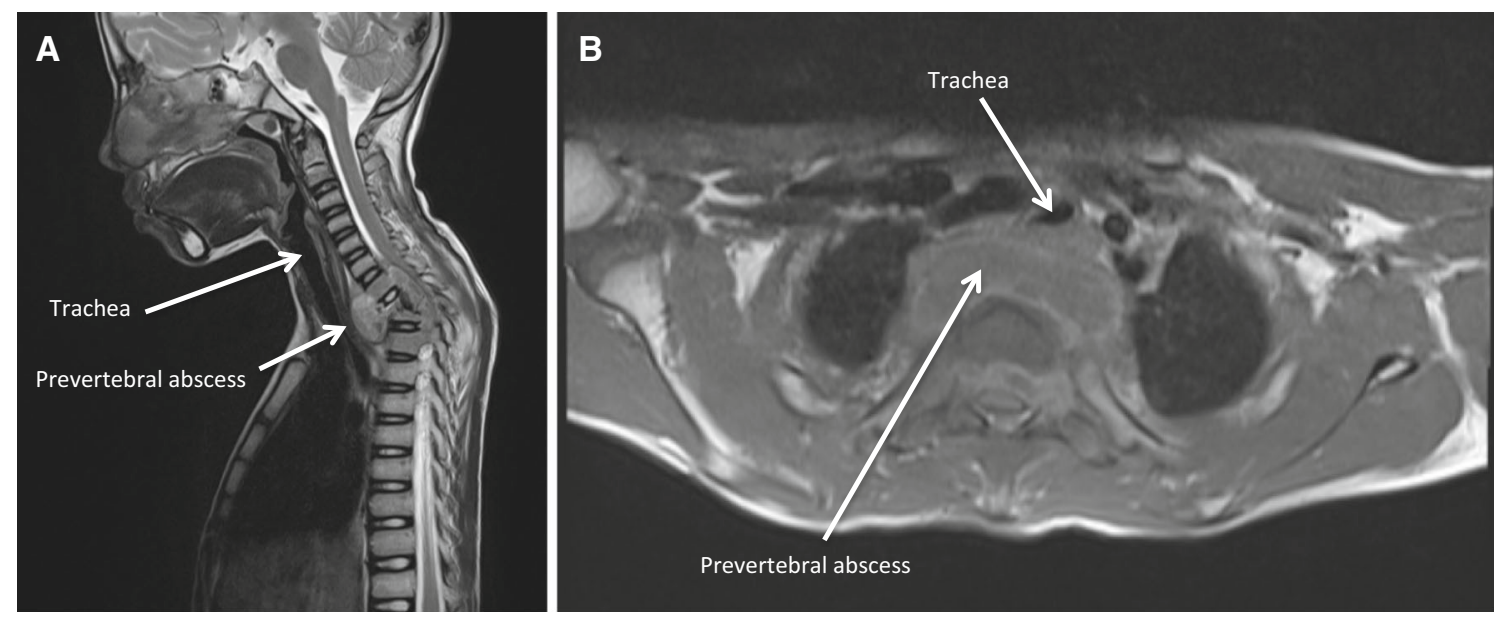

Figure Magnetic resonance imaging in the sagittal (A) and axial (B) planes of the cervical and thoracic spine in a patient with a prevertebral tuberculous abscess shows some distortion and compression of the trachea along with destruction of the underlying vertebrae

Conflicts of interest None declared.

Funding No external funding.

\section{References}

1. Rasouli MR, Mirkoohi M, Vaccaro AR, Yarandi KK, RahimiMovaghar $V$. Spinal tuberculosis: diagnosis and management. Asian Spine J 2012; 6: 294-308.
2. He M, Xи H, Zhao J, Wang Z. Anterior debridement, decompression, bone grafting, and instrumentation for lower cervical spine tuberculosis. Spine J 2014; 14: 619-27.

3. Pollard BA, El-Beheiry H. Pott's disease with unstable cervical spine, retropharyngeal cold abscess and progressive airway obstruction. Can J Anesth 1999; 46: 772-5. 